NBI-HE-96-37

July 16, 1996

\title{
The Branching of Graphs in 2-d Quantum Gravity
}

\author{
M. G. HARRIS \\ Niels Bohr Institute, Blegdamsvej 17, \\ DK-2100 Copenhagen, Denmark. \\ E-mail address: Martin.Harris@nbi.dk
}

\begin{abstract}
The branching ratio is calculated for three different models of $2 \mathrm{~d}$ gravity, using dynamical planar $\phi^{3}$ graphs. These models are pure gravity, the $D=-2$ Gaussian model coupled to gravity and the single spin Ising model coupled to gravity. The ratio gives a measure of how branched the graphs dominating the partition function are. Hence it can be used to estimate the location of the branched polymer phase for the multiple Ising model coupled to 2 d gravity.
\end{abstract}

\section{Introduction}

Many interesting results in the area of $2 \mathrm{~d}$ quantum gravity have been obtained by studying models of matter fields coupled to dynamical triangulations. However, all the exactly solved models have had central charges, $C$, of less than or equal to one (if one ignores models for which $C=\infty$ [1, 2]). Using conformal field theory it is possible to make predictions for the critical exponents in models of matter coupled to $2 \mathrm{~d}$ gravity [3, 佨, the so-called "KPZ formulae", but again these formulae are only valid for $C \leq 1$. The nature of this " $C=1$ barrier" remains something of a mystery. Many models of matter coupled to gravity have a tendency to degenerate into branched polymer configurations for large enough $C$. It is thus tempting to hypothesise that a branched polymer phase begins at $C=1$, causing the break down of the KPZ formulae here. Models in which multiple Ising spins are coupled to dynamical triangulations (or their dual $\phi^{3}$ graphs) provide a convenient way of testing this hypothesis; such a model with $p$ independent Ising spins on each vertex of the graph, has a central charge of $C=p / 2$ associated with it. However, Monte Carlo simulations for values of $p$ up to $p=16$ [5] have failed to show any convincing evidence of the existence of such a branched polymer ("BP") phase. Since 
there are reasons to believe that such a phase exists [1, 6], there are two possibilities to consider: firstly, that there exists an intermediate region between $C=1$ and the start of the branched polymer phase, or secondly, that the BP phase does indeed start at $C=1$ and that the $\mathrm{MC}$ simulations have for some reason failed to detect it. This paper aims to throw some light on the question by examining the branchedness of the graphs for various exactly solvable models.

In a recent paper [7] we studied correlation functions in a model of multiple Ising spins on a dynamical planar $\phi^{3}$ graph. In the paper, a function $B$ was defined which played a key rôle in the calculation of the critical exponents; $B$ gives a measure of how branched the $\phi^{3}$ graphs contributing to the partition function are and hereafter it will be called the "branching ratio". The branching ratio lies in the range 0 to 1 , and throughout the branched polymer phase $B$ equals one, when evaluated at the critical value of the cosmological constant. Thus the branching ratio gives a measure of how close to the BP phase we are, and by evaluating it for various models we will gain some indication of the extent of the branched polymer phase for the multiple Ising model coupled to gravity.

In this paper we calculate the branching ratio, which is defined in the next section, for three different models (all of which are defined in terms of planar $\phi^{3}$ graphs): pure gravity (section (3), the $D=-2$ Gaussian model coupled to gravity (section 1 ) and the single spin Ising model coupled to gravity (section 5). As might be expected the branching ratio increases with increasing central charge. In section 6 we conclude by using these results to make a prediction of the location of the branched polymer phase for the multiple Ising model coupled to gravity; it is estimated that there exists a BP phase for central charges above some value $\bar{C}$, where $\bar{C}$ lies in the range $1 \leq \bar{C} \lesssim 2.75$.

\section{The branching ratio}

In our previous paper [7] we used a simplified definition of distance rather than the usual geodesic distance. The geodesic distance between two vertices $A$ and $B$ of a $\phi^{3}$ graph is defined to be the shortest path along links, from $A$ to $B$, counting one unit of distance for each link traversed. However, in our definition of distance, only links separating two one-particle irreducible ("1PI") subgraphs are counted; figure 1 1 gives an example, each shaded circle represents a 1PI subgraph, hereafter referred to as a "blob".

Using this definition of distance it was possible to show that the number of blobs at a distance $r$ from the root, denoted $\langle n\rangle_{r}$, was given by $\langle n\rangle_{r}=B^{r}$, where $B$ is a function of the various parameters in the model.

For the case of a single Ising spin coupled to each vertex of a graph (using the set, $\mathcal{G}_{r}^{I}$, 
Figure 1: Measuring distances from the root blob

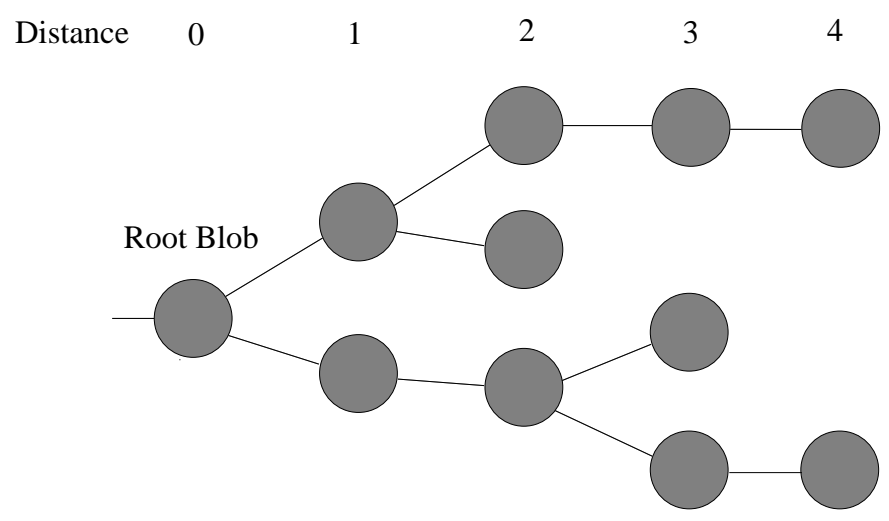

of all rooted planar $\phi^{3}$ graphs), we derived the following formula for $B$ [7],

$$
B(x, \beta)=\frac{x T}{1-2 x T}\left[1-3 x T+\frac{(1-x T)}{\mathcal{Z}^{I I}}\left(3 x^{\prime}\left(\frac{\partial \mathcal{Z}^{I I}}{\partial x^{\prime}}\right)_{\beta^{\prime}}+2 t^{\prime}\left(t^{\prime}-1\right)\left(\frac{\partial \mathcal{Z}^{I I}}{\partial t^{\prime}}\right)_{x^{\prime}}\right)\right],
$$

where $T \equiv \mathcal{Z}^{I}(x, \beta)$. The grand canonical partition function for the single spin Ising model on the set of graphs $\mathcal{G}_{r}^{I}$ is defined by

$$
\mathcal{Z}^{I}(x, \beta)=\sum_{G \in \mathcal{G}_{r}^{I}} x^{N} \frac{1}{2^{N}} \sum_{\{S\}} \prod_{<i j>}\left[1+t S_{i} S_{j}\right],
$$

where $S_{i}$ is the spin on vertex $i$ and $t=\tanh \beta$. This will be referred to as model I. The coupling constant $\beta$ is the inverse temperature of the model and each vertex in the graph is weighted with a factor of $x$. The first summation is over graphs $G$ in the set $\mathcal{G}_{r}^{I}$, where $N$ is the number of vertices in $G$, the second summation is over all spin configurations and the product is over nearest neighbour pairs. Note that there is no spin at the end of the root and hence the root link is not included in this product. The partition function $\mathcal{Z}^{I I} \equiv \mathcal{Z}^{I I}\left(x^{\prime}, \beta^{\prime}\right)$ is defined as in (2), but using the set of planar rooted 1PI $\phi^{3}$ graphs, $\mathcal{G}_{r}^{I I}$; this will be called model II. The renormalized coupling constants $t^{\prime}=\tanh \beta^{\prime}$ and $x^{\prime}$ are given by

$$
x^{\prime}=x(1-2 x T)^{-\frac{3}{2}}, \quad \quad t^{\prime}=t \frac{(1-2 x T)}{(1-2 x T t)}
$$

and the following equation which relates $\mathcal{Z}^{I}$ and $\mathcal{Z}^{I I}$ was derived in our paper [7],

$$
T=\sqrt{1-2 x T} \mathcal{Z}^{I I}\left(x^{\prime}, \beta^{\prime}\right)+x T^{2} .
$$




\section{$3 \quad$ Pure gravity}

In the case of pure gravity (that is, equation (2) with $\beta=0$ ) equation (11) reduces to

$$
B(x)=\frac{x T}{1-2 x T}\left[1-3 x T+3(1-x T) \frac{x^{\prime}}{\mathcal{Z}^{I I}} \frac{\partial \mathcal{Z}^{I I}}{\partial x^{\prime}}\right] .
$$

We are interested in evaluating this at the critical value of $x$ for model $\mathrm{I}$, which is denoted by $x_{c}^{I}$. From reference [8], $x_{c}^{I}=1 /\left(2.3^{\frac{3}{4}}\right)$ and $T\left(x_{c}^{I}\right)=3^{\frac{3}{4}}(1-\sqrt{3} / 2)$. The corresponding value of $x^{\prime}$ is $x_{c}^{I I}=\sqrt{\frac{2}{27}}$ and in reference [7] it was shown that

$$
\left.\frac{x^{\prime}}{\mathcal{Z}^{I I}} \frac{\partial \mathcal{Z}^{I I}}{\partial x^{\prime}}\right|_{x^{\prime}=x_{c}^{I I}}=\frac{5}{3}
$$

Thus writing $B_{c}$ for $B\left(x_{c}^{I}\right)$, we have that the branching ratio at the critical value of $x$ is

$$
B_{c}=1-\frac{1}{\sqrt{3}} \approx 0.4226
$$

for pure gravity, with rooted planar $\phi^{3}$ graphs; this model, of course, corresponds to a central charge of zero.

\section{Gaussian model}

In this section we will derive $B_{c}$ for the Gaussian model in $D=-2$ dimensions, which has a central charge of -2 . For this choice of $D$ the partition function has an especially simple form [9],

$$
\mathcal{Z}_{g}^{I}(x)=\sum_{G \in \mathcal{G}_{r}^{I}} \sum_{T \in \mathcal{T}_{G}} x^{N}
$$

Again the set $\mathcal{G}_{r}^{I}$ of all rooted planar $\phi^{3}$ graphs is being used and in addition there is a summation over the trees $T$ in the set of all trees spanning $G$; this set is denoted by $\mathcal{T}_{G}$.

The partition function has an integral formulation [10],

$$
\mathcal{Z}_{g}^{I}(x)=\frac{1}{x \pi} \int_{-1}^{1} d t \sqrt{1-t^{2}}(1-\sqrt{1-8 x t})
$$

which has a critical value of $x$ given by $x_{c}^{I}=\frac{1}{8}$. At this critical value,

$$
\begin{aligned}
& \mathcal{Z}_{g}^{I}\left(x_{c}^{I}\right)=4\left(1-\frac{32 \sqrt{2}}{15 \pi}\right) \\
& \left.\frac{d \mathcal{Z}_{g}^{I}}{d x}\right|_{x_{c}^{I}}=32\left(\frac{12 \sqrt{2}}{5 \pi}-1\right)
\end{aligned}
$$


Figure 2: $T=f(T)$

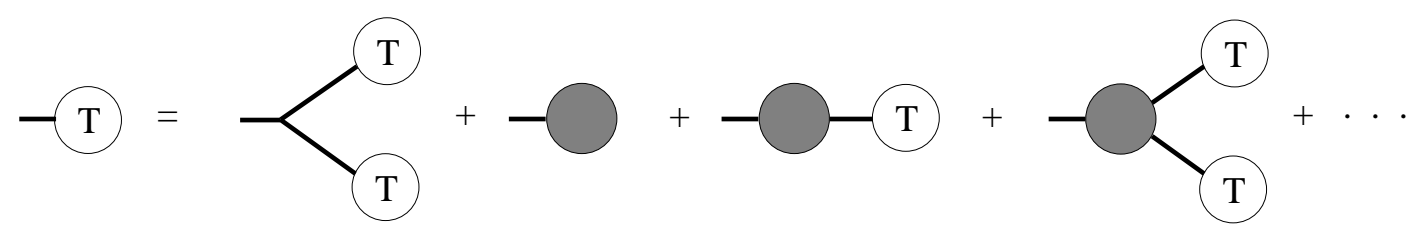

and $\frac{d^{2} \mathcal{Z}_{g}^{I}}{d x^{2}}$ diverges.

A formula for $B(x)$ in this model can be obtained by using a derivation similar to that for the multiple Ising model [7]. Define a function, $f(y)$, which takes a rooted 1PI blob (both graphs and spanning trees are being summed over in the blob) and glues an arbitrary number of legs, each weighted with a factor $y$, on to the blob. Again we define $T \equiv \mathcal{Z}_{g}^{I}(x)$ to save writing and have that $T=f(T)$, which is illustrated in figure 2. To derive a formula for $f(y)$ we need to consider the effect of adding an arbitrary number of legs to a link. For a given graph $G$ and spanning tree $T$ there are two different types of link, those that form part of the spanning tree, which will be referred to as "black" links (drawn as thick lines), and those which are not part of the spanning tree (these are called "white" and drawn as thin lines). Adding legs to a black link changes the contribution it makes to the partition function (fig $3 \mathrm{a}$ ):

$$
1 \rightarrow 1+2 x y+(2 x y)^{2}+\cdots=\frac{1}{1-2 x y} .
$$

For each leg added there is a weight of $y$, a weight of $x$ for the extra vertex and a factor of 2 , since the leg can be hung from the link in one of two directions; this accounts for the factors of $2 x y$.

Similarly for white links there is the change (fig $3 \mathrm{~b})$ :

$$
1 \rightarrow 1+2(2 x y)+3(2 x y)^{2}+4(2 x y)^{3}+\cdots=\frac{1}{(1-2 x y)^{2}} .
$$

In this case one must also take into account the different ways of connecting the new legs to the existing spanning tree.

The partition function for rooted 1PI blobs is defined by

$$
\mathcal{Z}_{g}^{I I}(x)=\sum_{G \in \mathcal{G}_{r}^{I I}} \sum_{T \in \mathcal{T}_{G}} x^{N}
$$

Adding legs to this changes the partition function for the blob to

$$
\sum_{G \in \mathcal{G}_{r}^{I I}} \sum_{T \in \mathcal{T}_{G}} x^{N}(1-2 x y)^{-\left(L_{B}+2 L_{W}\right)},
$$


Figure 3: Renormalization of links: (a) Black, (b) White

(a)

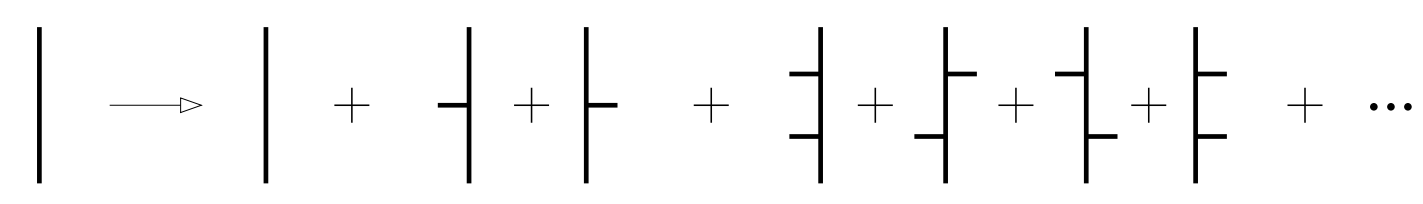

(b)

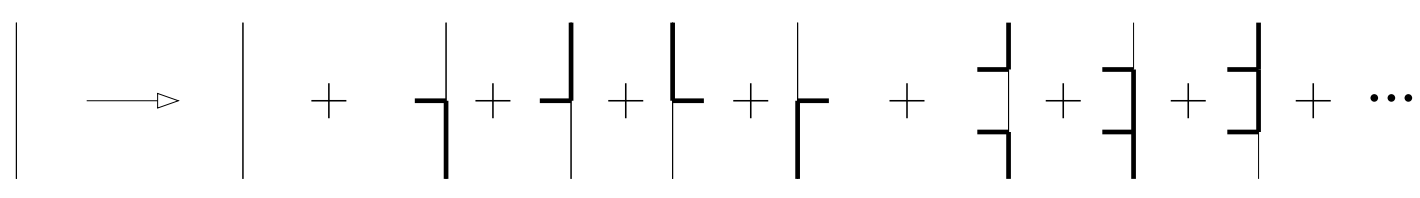

where the number of black links is $L_{B}=N-1$ and the number of white links is $L_{W}=$ $\frac{1}{2}(N+1)$. Thus $L_{B}+2 L_{W}=2 N$ and hence

$$
f(y)=x y^{2}+\mathcal{Z}_{g}^{I I}\left(x(1-2 x y)^{-2}\right) .
$$

It is convenient to define $x^{\prime}=x(1-2 x T)^{-2}$. Note that at $x=x_{c}^{I}$, we have $x^{\prime}=x_{c}^{I I} \equiv$ $\left(\frac{15 \pi}{128}\right)^{2}$, which agrees with the result in reference [11. As before $B$ is given by

$$
B=\left.\frac{\partial f}{\partial y}\right|_{y=T},
$$

so that, with $\mathcal{Z}_{g}^{I I} \equiv \mathcal{Z}_{g}^{I I}\left(x^{\prime}\right)$,

$$
B(x)=2 x\left[T+\frac{d \mathcal{Z}_{g}^{I I}}{d x^{\prime}} \frac{2 x^{\prime}}{1-2 x T}\right] .
$$

Differentiating the equation $T=f(T)$ with respect to $x^{\prime}$ yields an expression for $\frac{d \mathcal{Z}_{g}^{I I}}{d x^{\prime}}$ and then evaluating (18) at $x=x_{c}^{I}$ gives

$$
B_{c}=1-\frac{3 \pi}{8 \sqrt{2}} \approx 0.1670
$$

for the $D=-2$ Gaussian model.

\section{$5 \quad$ Ising model}

Finally, we will calculate the branching ratio for the Ising model on planar $\phi^{3}$ graphs with a single spin per vertex; the matter in this model has a central charge of $\frac{1}{2}$. To use equation (1) we need the partition functions for both model I and model II, which we 
obtain by solving the following two-matrix model,

$$
\begin{aligned}
\mathcal{Z}=e^{M^{2} F(g, c, \lambda)} & =\int \mathcal{D} A \mathcal{D} B \exp \operatorname{Tr}\left[-\frac{1}{2}\left(A^{2}+B^{2}\right)+c A B\right. \\
& \left.+\frac{1}{3} \frac{g}{\sqrt{M}}\left(A^{3}+B^{3}\right)+\lambda \sqrt{M}(A+B)\right]
\end{aligned}
$$

where $A$ and $B$ are $M \times M$ Hermitian matrices and we wish to take the planar limit in which $M \rightarrow \infty$. Each vertex is weighted with $g$ and the coupling constant $c$ equals $e^{-2 \beta}$. In the case $\lambda=0, F$ will give the model I partition function up to various factors; this has been solved in references [12, 13]. Alternatively by choosing $\lambda$ so that graphs with tadpoles are cancelled, we gain the partition function for model II.

This matrix model can be solved using orthogonal polynomials [14]. In the planar limit and dropping the constant term, we have

$$
\begin{gathered}
F(g, c, \lambda)=\frac{1}{2} \ln \left(\frac{1}{g^{2}} z(u=1)\right)+\int_{0}^{1}\left(\frac{1}{2} u^{2}-u\right) \frac{1}{z} \frac{d z}{d u} d u+\frac{3}{4}+\frac{1}{2} \ln \left(1-c^{2}\right) \\
-\frac{1}{3}\left(\frac{1}{2 g}\right)^{2}\left(1+\rho_{0}\right)\left[(1-c)\left(1+\rho_{0}\right)-8 \lambda g\right]
\end{gathered}
$$

where

$$
\begin{gathered}
u g^{2}=z\left(\rho^{2}-c^{2}\right)+2 c z^{2}, \\
z=\frac{1}{8 \rho}[(\rho+1)(\rho-1+2 c)+4 \lambda g]
\end{gathered}
$$

and $\rho_{0}$ is $\rho$ evaluated at $u=0$ (i.e. at $z=0$ ),

$$
\rho_{0}=-c-\sqrt{(1-c)^{2}-4 \lambda g} .
$$

After some considerable algebra we obtain,

$$
g\left(\frac{\partial F}{\partial \lambda}\right)_{g, c}=1+\rho_{1}-\frac{2}{g^{2}} z_{1}^{2} \rho_{1}\left(\rho_{1}-c\right)
$$

where $\rho_{1}=\rho(u=1)$ and $z_{1}=z(u=1)$. Note that $F$ generates unrooted (i.e. vacuum) graphs and that by differentiating with respect to $\lambda$ we generate rooted graphs instead.

Now, the model I partition function that we want to calculate is related to that generated from the matrix model by

$$
x T \equiv x \mathcal{Z}^{I}(x, \beta)=\left.\frac{1}{2}\left(\frac{g}{1-c}\right) \frac{\partial F}{\partial \lambda}\right|_{\lambda=0} .
$$

Thus dropping the subscripts,

$$
x T=\frac{1}{2} \frac{(1+\rho)}{(1-c)}\left[1-\frac{\rho(\rho-c)(\rho-\alpha)}{\left(4 \rho^{3}+c \rho^{2}-2 c^{2} \rho-c \alpha\right)}\right],
$$


where $c=e^{-2 \beta}, \alpha=1-2 c$,

$$
\begin{gathered}
z=\frac{1}{8 \rho}(\rho+1)(\rho-\alpha), \\
g^{2}=z\left(\rho^{2}-c^{2}\right)+2 c z^{2}=2(1-c)^{3} x^{2} .
\end{gathered}
$$

At $x_{c}^{I}$, which is given by $\left(\frac{\partial g}{\partial \rho}\right)_{c}=0$,

$$
\begin{aligned}
\rho=-\sqrt{\frac{1}{3}(1-2 c)} & \left(\text { for } \beta>\beta^{*}\right), \\
2 \rho^{3}+3 c \rho^{2}+c(1-2 c)=0 & \left(\text { for } \beta<\beta^{*}\right) .
\end{aligned}
$$

There is a third order phase transition in model I, at

$$
c^{*}=\frac{1}{27}(2 \sqrt{7}-1),
$$

between a magnetized and an unmagnetized phase.

For model II we need to choose $\lambda=\bar{\lambda}$ such that $\left.\frac{\partial F}{\partial \lambda}\right|_{\bar{\lambda}}$ vanishes. This gives us,

$$
g^{2}=\frac{\rho(1+\rho)(\rho+c)^{2}(\rho-c)^{3}}{2\left(\rho^{2}-2 \rho c-c\right)^{2}}
$$

and

$$
\bar{\lambda} g=\frac{1}{4} \frac{(1+\rho)}{\left(\rho^{2}-2 \rho c-c\right)}\left(3 \rho^{3}+\rho^{2}-\rho c-c(1-2 c)\right) .
$$

At the critical value of $x$, denoted $x_{c}^{I I}$,

$$
\begin{aligned}
3 \rho^{2}+2 \rho+c & =0 & & \left(\text { for } \beta>\beta^{*}\right), \\
\rho^{3}-3 c \rho^{2}-3 c \rho+c^{2} & =0 & & \left(\text { for } \beta<\beta^{*}\right) .
\end{aligned}
$$

There is a third order phase transition at $c^{*}=\frac{5}{27}$, and the model II partition function is

$$
x \mathcal{Z}^{I I}(x, \beta)=-\frac{\bar{\lambda} g}{(1-c)^{2}} .
$$

Defining

$$
E(x, \beta) \equiv \frac{1}{\mathcal{Z}^{I I}(x, \beta)}\left[3 x\left(\frac{\partial \mathcal{Z}^{I I}}{\partial x}\right)_{\beta}+2 t(t-1)\left(\frac{\partial \mathcal{Z}^{I I}}{\partial t}\right)_{x}\right],
$$

we have from equations (33), (34) and (37) that

$$
E(x, \beta)=4 c-3+\frac{4(1-c)\left(3 \rho^{3}+c^{2}\right)}{\left(3 \rho^{3}+\rho^{2}-\rho c-c(1-2 c)\right)} .
$$

Then

$$
B(x, \beta)=\frac{x T}{1-2 x T}\left[1-3 x T+(1-x T) E\left(x^{\prime}, \beta^{\prime}\right)\right]
$$


where $E$ is given by the previous equation, $x^{\prime}$ and $\beta^{\prime}$ by (3) and $x T$ is given by (27). Evaluating this at the critical value of $x$ gives $B_{c}(\beta) \equiv B\left(x_{c}^{I}(\beta), \beta\right)$; figure 4 gives a plot of $B_{c}$ against $c=e^{-2 \beta}$. The branching ratio is a maximum at the phase transition and one can show that the branching ratio there is

$$
B_{c}\left(\beta^{*}\right)=1-\frac{5}{4 \sqrt{7}} \approx 0.5275
$$

Note that as expected both model I and model II have $\gamma_{s t r}=-\frac{1}{2}$ away from the transition and $\gamma_{s t r}^{*}=-\frac{1}{3}$ at the critical point.

Figure 4: Graph of the branching ratio, $B_{c}(\beta)$, against $c=e^{-2 \beta}$ for the single spin Ising model coupled to $2 \mathrm{~d}$ gravity.

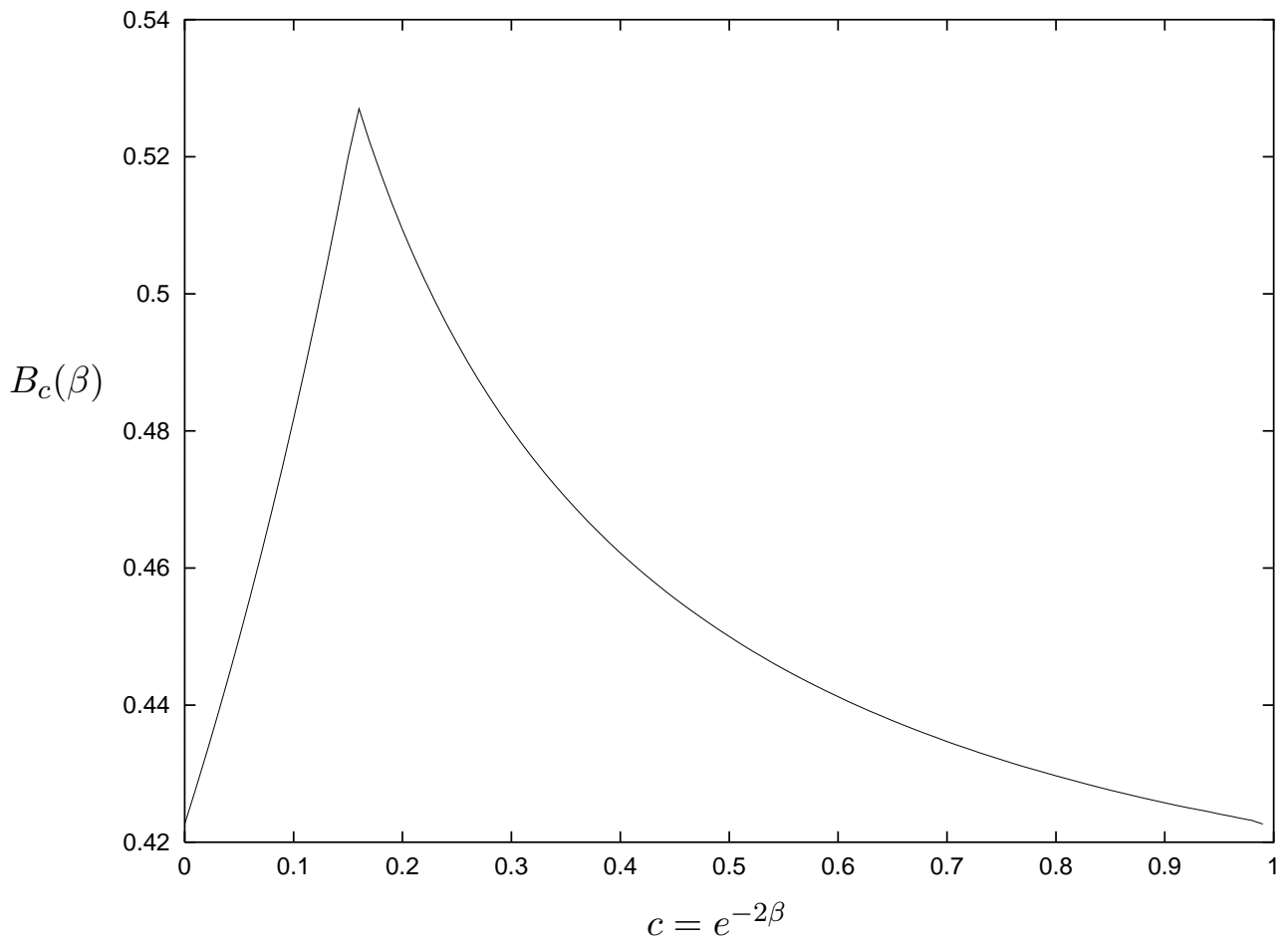

\section{Conclusion}

In this paper we have calculated the branching ratio for three different models coupled to $2 \mathrm{~d}$ gravity, in the form of rooted planar $\phi^{3}$ graphs. In figure 5 are plotted the values of $B_{c}$ 
Figure 5: Graph of the branching ratio, $B_{c}$, against the central charge, $C$, for various models coupled to $2 \mathrm{~d}$ gravity.

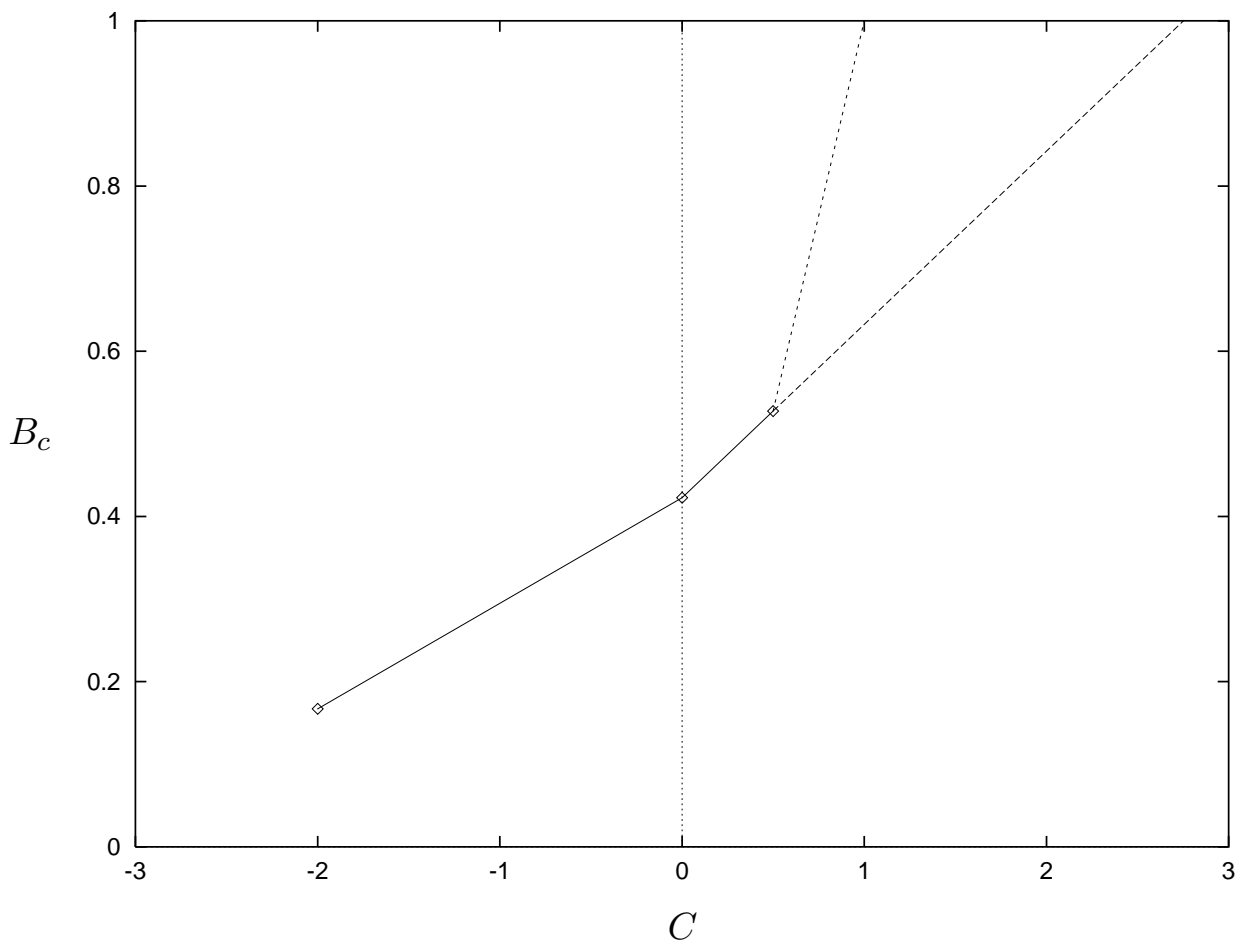

against the central charge, $C$; for the case of the single spin Ising model we used $B_{c}\left(\beta^{*}\right)$, since the graphs are most branched at the phase transition. We see that the graphs tend to become more branched as the central charge increases. This is not unexpected since many models coupled to gravity degenerate to branched polymers for large enough central charge. One should perhaps note at this point that $B_{c}$ is not a universal function of the central charge, so that different models with $C=-2$ will in general have different branching ratios. Thus the data point for the Gaussian model is probably not very reliable when trying to determine the behaviour of the multiple Ising model. For this reason we extrapolate using only the last two data points and estimate that $B_{c}=1$ at $C=2.75$ (the extrapolation is the right-most dotted line in figure 5). This is likely to be an overestimate since the graph seems to be curving upwards with $C$. Even if we ignore the data point for the Gaussian model we know that for $C \rightarrow-\infty, B_{c}$ must tend to a finite non-negative value and in this limit one might guess that $B_{c} \rightarrow 0$; so that it seems likely that the graph will be curving upwards in the region of interest. In our previous paper [7] it was shown 
that regions of the phase diagram with $B_{c}=1$ correspond to the branched polymer phase

$\left(\gamma_{s t r}=\frac{1}{2}\right)$ and its boundary $\left(\gamma_{s t r} \geq 0\right)$. It is known that there is no branched polymer phase for $C<1$ and thus the left-most dotted line indicates an approximate upper limit on possible values of $B_{c}$. So we conclude that the curve of $B_{c}$ against $C$ probably lies within the region bounded by the two dotted lines and hence that there exists a branched polymer phase for central charges greater than some value $\bar{C}$ where $1 \leq \bar{C} \lesssim 2.75$. Note that this estimate for $\bar{C}$ is lower than previous cruder estimates [6, 15]. Our result is still consistent with the hypothesis $\bar{C}=1$, although it is not possible to rule out the existence of an intermediate region. Even though we are unable to distinguish between these two possibilities it seems likely that any intermediate region which exists would be quite narrow. Further work will be required in order to determine more fully the phase diagram for the multiple Ising model coupled to gravity and to understand the nature of the $C=1$ barrier in general.

\section{Acknowledgements}

The author would like to acknowledge the support of the Royal Society through their European Science Exchange Programme.

\section{References}

[1] M.Wexler, Nucl. Phys. B410 (1993) 377, hep-th/9305041;

M.Wexler, Mod. Phys. Lett. A8 (1993) 2703, hep-th/9307120.

[2] J.Ambjørn, B.Durhuus and T.Jónsson, Mod. Phys. Lett. A9 (1994) 1221, hepth/9401137.

[3] V.G.Knizhnik, A.M.Polyakov and A.B. Zamolodchikov, Mod. Phys. Lett. A3 (1988) 819.

[4] J.Distler and H.Kawai, Nucl. Phys. B321 (1989) 509;

F.David, Mod. Phys. Lett. A3 (1988) 1651.

[5] C.F.Baillie and D.A.Johnston, Phys. Lett. B286 (1992) 44, hep-lat/9204003;

S.M.Catterall, J.B.Kogut and R.L.Renken, Phys. Lett. B292 (1992) 277, heplat/9206021;

J.Ambjørn, B.Durhuus, T.Jónsson and G. Thorleifsson, Nucl. Phys. B398 (1993) 568, hep-th/9208030;

M.Bowick, M.Falcioni, G.Harris and E.Marinari, Nucl. Phys. B419 [FS] (1994) 665, hep-th/9310136; 
J.Ambjørn and G.Thorleifsson, Phys. Lett. B323 (1994) 7, hep-th/9312157;

J.-P.Kownacki and A.Krzywicki, Phys. Rev. D50 (1994) 5329, hep-th/9401143.

[6] M.G.Harris and J.F.Wheater, Nucl. Phys. B427 (1994) 111, hep-th/9404174.

[7] M.G.Harris and J.Ambjørn, "Correlation functions in the multiple Ising model coupled to gravity", to appear in Nucl. Phys. B, hep-th/9602028.

[8] E.Brézin, C.Itzykson, G.Parisi and J.B.Zuber, Commun. Math. Phys. 59 (1978) 35.

[9] D.V.Boulatov, V.A.Kazakov, I.K.Kostov and A.A.Migdal, Nucl. Phys. B275 [FS17] (1986) 641.

[10] V.A.Kazakov, I.K.Kostov and A.A.Migdal, Phys. Lett. B157 (1985) 295.

[11] F.David, Phys. Lett. B159 (1985) 303.

[12] D.V.Boulatov and V.A.Kazakov, Phys. Lett. B186 (1987) 379.

[13] Z.Burda and J.Jurkiewicz, Acta Phys. Pol. B120 (1989) 949.

[14] M.L.Mehta, Commun. Math. Phys. 79 (1981) 327.

[15] M.G.Harris, Mod. Phys. Lett. A11 (1996) 553, hep-th/9502036. 